\title{
Knockdown of homeobox containing 1 increases the radiosensitivity of cervical cancer cells through telomere shortening
}

\author{
SHULIANG ZHOU ${ }^{1,2^{*}}$, YOUDE XIAO $^{1,2^{*}}$, YAFEI ZHUANG ${ }^{1,2}$, YINYIN LIU $^{1,2}$, HONG ZHAO $^{1,2}$, \\ HUI YANG ${ }^{1,2}$, CONGHUA XIE ${ }^{1,2}$, FUXIANG ZHOU ${ }^{1,2}$ and YUNFENG ZHOU ${ }^{1,2}$ \\ ${ }^{1}$ Department of Radiation and Medical Oncology, Zhongnan Hospital of Wuhan University; \\ ${ }^{2}$ Hubei Cancer Clinical Study Center, Hubei Key Laboratory of Tumor Biological Behaviors, \\ Zhongnan Hospital of Wuhan University, Wuhan, Hubei 430071, P.R. China
}

Received November 4, 2016; Accepted May 23, 2017

DOI: $10.3892 /$ or.2017.5707

\begin{abstract}
Homeobox containing 1 (HMBOX1) modulates telomere length in various types of tumor cells by binding to double-stranded telomeric DNA. There is a negative correlation between telomere length and radiosensitivity in tumor cells. In the present study, we aimed to investigate the relationship among HMBOX1, telomere and radiosensitivity in cervical cancer cells. Lentivirus-based shRNAs were used to establish stable transfected cell lines in which protein and mRNA levels of HMBOX1 were notably decreased. Knockdown of HMBOX1 increased the radiosensitivity of HeLa and C33A cells. TERT protein was also decreased while HMBOX1 was downregulated. Knockdown of HMBOX1 shortened telomere length in the HeLa cells, while TERT overexpression rescued telomere shortening in the HeLa-HMBOX1 cells. Knockdown of HMBOX1 increased the apoptosis rate, decreased radiationinduced DNA damage foci, and inhibited the expression of ATM, ATR, p-ATM, p-ATR and BRCA1 in the homologous recombination repair pathway. Our data suggest a possible role of HMBOX1 in regulating radiosensitivity in cervical cancer cells. Moreover, HMBOX1 may be a potential factor in the radiotherapy of cervical cancer.
\end{abstract}

Correspondence to: Professor Fuxiang Zhou or Professor Yunfeng Zhou, Department of Radiation and Medical Oncology, Zhongnan Hospital, Wuhan University, Wuhan, Hubei 430071, P.R. China

E-mail: happyzhoufx@sina.com

E-mail: yfzhouwhu@163.com

*Contributed equally

Abbreviations: DSBs, DNA double-stranded breaks; $\gamma \mathrm{H} 2 \mathrm{AX}$, phosphorylated histone $\mathrm{H} 2 \mathrm{AX}$; IR, ionizing radiation; ATM, ataxia telangiectasia-mutated; ATR, ataxia telangiectasia rad3-related

Key words: HMBOX1, cervical cancer, radiosensitivity, telomere length, DNA damage, apoptosis

\section{Introduction}

Cervical cancer is the most common malignant tumor of the female reproductive system. It is the second most commonly diagnosed cancer and the third leading cause of cancerrelated death among females in developing countries (1). Radiotherapy is the major treatment for cervical cancer. For patients with early-stage disease, radiotherapy and surgery alone have equal effects. In recent years, chemoradiotherapy has gradually become a new treatment pattern for patients with locally advanced disease. It has been demonstrated that radiotherapy plays a crucial role in the treatment of cervical cancer. However, patients who suffer pelvic recurrence account for $\sim 70 \%$ of the cases of radiotherapy failure (2). Thus, the identification of new factors that influence radiosensitivity has important significance in cervical cancer treatment.

Homeobox containing 1 (HMBOX1), a new member of the homeobox genes, was identified and isolated from the human pancreatic cDNA library. HMBOX1 was described as a transcription repressor (3). In the ALT (alternative lengthening of telomeres) cell line WI38-VA13 and telomerase-positive HeLa cells, HMBOX1 was identified as a double-stranded telomeric DNA binding protein (4) and acted as a positive regulator of telomere length (5). In U2OS (ALT cells), HMBOX1 was found to modulate telomere maintenance without influencing telomere length (6). However, the further function of HMBOX1 is not fully clear.

Telomeres, nucleoprotein complexes at the end of eukaryotic chromosomes, are composed of 5'-TTAGGG-3' repeats and are maintained by telomerase. Telomeres contain protein complexes and shelterin, and play a crucial role in protecting chromosome ends from DNA damage. Moreover, telomere length may serve as a target in predicting the individual radiosensitivity of patients with cancers (7-11).

However, to date, the relationship between HMBOX1 and radiosensitivity remains unclear. Therefore, the present study aimed to investigate whether HMBOX1 modulates radiosensitivity in cervical cancer cells and to explore the potential mechanism. 


\section{Materials and methods}

Cell lines and cell culture. The human cervical cancer cell lines (HeLa and C33A) were maintained by the Key Laboratory of Tumor Biological Behavior of Hubei Province. All cells were cultured in minimum essential medium (MEM) (HyClone, Logan, UT, USA) supplemented with $10 \%$ fetal bovine serum (Gibco, Grand Island, NY, USA), $100 \mathrm{U} / \mathrm{ml}$ penicillin and $100 \mu \mathrm{g} / \mathrm{ml}$ streptomycin (BioSharp, Hefei, China) in $5 \% \mathrm{CO}_{2}$ at $37^{\circ} \mathrm{C}$.

Transfections, lentiviral shRNA and plasmids. HeLa and C33A cells were transfected with lentiviral shRNA (GenePharma, Shanghai, China). The sequences of shRNA were: shGFP, 5'-GTGGTACCAGCATCAGCCTT-3', and shHMBOX1, 5'-GGACCTAGATGTAGATGAT-3'. Forty-eight hours after transfection, the cells were treated with $1 \mu \mathrm{g} / \mathrm{ml}$ puromycin for 2 weeks. The stable transfected cell lines were named as HeLa-NC, C33A-NC, HeLa-HMBOX1 and C33AHMBOX1, respectively. HeLa-HMBOX1 cells were transfected with pHBLV-CMVIE-ZsGreen-TERT using Lipofectamine 2000 (Invitrogen, Carlsbad, CA, USA).

Western blot analysis and real-time PCR. Radioimmunoprecipitationassay (RIPA)lysisbufferand phenylmethylsulfonyl fluoride (PMSF) were used to extract total protein from the cultured cells. Western blotting was performed as previously described (12). The following antibodies were used: GAPDH (10494-1-AP), tubulin (11224-1-AP), HMBOX1 (16123-1-AP) (all from Proteintech, Wuhan, China), TERT (H231; Santa Cruz Biotechnology, Santa Cruz, CA, USA), BRCA1 (206491-AP) (Proteintech), ATM (2873), ATR (2790), phospho-ATM (13050), phospho-ATR (2853), caspase-3 (9662) (all from Cell Signaling Technology, Inc., Danvers, MA, USA) and $\gamma \mathrm{H} 2 \mathrm{AX}$ (ab11174; Abcam, Cambridge, MA, USA).

Real-time PCR was used to detect the relative mRNA level and telomere length. Total RNA was extracted from cultured cells using TRIzol reagent (BioSharp). cDNA was synthesized using the PrimeScript ${ }^{\mathrm{TM}}$ II First Strand cDNA Synthesis kit (Takara, Dalian, China). Total DNA was extracted using the E.Z.N.A. Tissue DNA kit (Omega Bio-Tek, Inc., Norcross, RA, USA). Then, cDNA and DNA were amplified using the CFX96 Real-Time PCR Detection System (Bio-Rad, Hercules, CA, USA) by SYBR Premix Ex Taq ${ }^{\mathrm{TM}}$ (Takara). The protocols of GAPDH, HMBOX1 and TERT were performed as follows: pre-degeneration at $95^{\circ} \mathrm{C}$ for $5 \mathrm{sec}, 40$ cycles of $95^{\circ} \mathrm{C}$ for $15 \mathrm{sec}$, $60^{\circ} \mathrm{C}$ for $30 \mathrm{sec}$.

36B4 and telomere primers were used to detect relative telomere length as follows (13): pre-degeneration at $95^{\circ} \mathrm{C}$ for $10 \mathrm{~min}, 40$ cycles of $95^{\circ} \mathrm{C}$ for $15 \mathrm{sec}, 54^{\circ} \mathrm{C}$ for $2 \mathrm{~min}$. All primer sequences were synthesized by Tsingke Biological Technology (Wuhan, China): GAPDH (forward, 5'-TGGAAGGACTCA TGACCACA-3' and reverse, 5'-TTCAGCTCAGGGATGAC CTT-3'); HMBOX1 (forward, 5'-CTTCAGCGACTTCGGC GTA-3' and reverse, 5'-ATCATAACTGTTGCTAGGTGA CG-3'); TERT (forward, 5'-CATTTCATCAGCAAGTTTGG AAG-3' and reverse, 5'-TTTCAGGATGGAGTAGCAGA GG-3'); tel (tel1, 5'-GGTTTTTGAGGGTGAGGGTGAG GGTGAGGGTGAGGGT-3' and tel2, 5'-TCCCGACTAT СССТАТСССТАТСССТАТСССТАТСССТА-3'); 36B4
(36B4u, 5'-CAGCAAGTGGGAAGGTGTAATCC-3' and 36B4d, 5'-CCCATTCTATCATCAACGGGTACAA-3').

Colony formation assay. Cells were harvested from culture flasks and plated into 6-well plates at appropriate dilutions, and with different viable cells at 100, 100, 200, 400, 800, 1,000 and 2,000/well of HeLa-NC and HeLa-HMBOX1 cells, respectively. Each plate was irradiated with $0,1,2,4,6,8$, 10 Gy of X-rays after $12 \mathrm{~h}$, respectively. After culturing for 14 days, the cells were fixed and stained using paraformaldehyde and Giemsa; clones were considered to be viable cells when containing at least 50 cells. Different cell lines have different plating efficiencies after IR exposure. C33A cells have a higher intrinsic radiosensitivity compared with HeLa, thus C33A-NC and C33A-HMBOX1 cells were plated with viable cells at 100,150,300,1,000,6000,20,000 and 100,000/ well, respectively. Other conditions were the same as for the HeLa cells $(12,14,15)$. Data of the survival fraction were fitted into multi-target and single-hit models, and survival curves were drawn using GraphPad Prism 5.0 software.

Apoptosis assay. Apoptosis was assessed in the cells with or without 6 Gy X-ray exposure, and then cultured for $48 \mathrm{~h}$. Apoptosis assay was performed using an Annexin V-PE and 7-AAD apoptosis analysis kit (Sungene Biotech Co., Ltd., Tianjin, China) according to the protocol, and then analyzed by a flow cytometer (Beckman Coulter, Brea, CA, USA).

Immunofluorescence. $\gamma \mathrm{H} 2 \mathrm{AX}$ was identified as a molecular marker of DSBs, detected by immunofluorescence. Cells were divided into 4 groups: HeLa-NC, HeLa-HMBOX1, HeLa-NC + 4Gy and HeLa-HMBOX1 + 4Gy. Thirty minutes later, the cells with and without IR exposure were fixed with paraformaldehyde for $15 \mathrm{~min}$, permeabilized with $0.2 \%$ Triton X-100 for $20 \mathrm{~min}$, blocked with goat serum for $2 \mathrm{~h}$ and incubated with the primary antibody overnight at $4^{\circ} \mathrm{C}$, and then washed with phosphate-buffered saline (PBS) and incubated with the secondary antibody for $2 \mathrm{~h}$ away from light at room temperature. Nuclei were stained with DAPI (Sigma, St. Louis, MO, USA). Images were obtained using a confocal microscope (Leica Microsystems GmbH, Wetzlar, Germany).

Statistical analysis. All data were obtained from 3 independent experiments. SPSS 17.0 and GraphPad Prism 5.0 were used to analyze data. Data are expressed as mean \pm SD. Independent samples t-test was used for the comparison of data, and $\mathrm{P}<0.05$ was considered to indicate a statistically significant difference.

\section{Results}

Efficiency of HMBOX1 knockdown in cervical cancer cells. The expression of protein and mRNA was assessed using western blotting and real-time PCR, respectively. As shown in Fig. 1A and B, the expression of HMBOX1 was obviously decreased both at the protein and mRNA level in the HeLa cells. The results for the $\mathrm{C} 33 \mathrm{~A}$ cells were similar as those for the HeLa cells. These results indicated that we successfully established stable transfected cell lines of HeLa and C33A. 

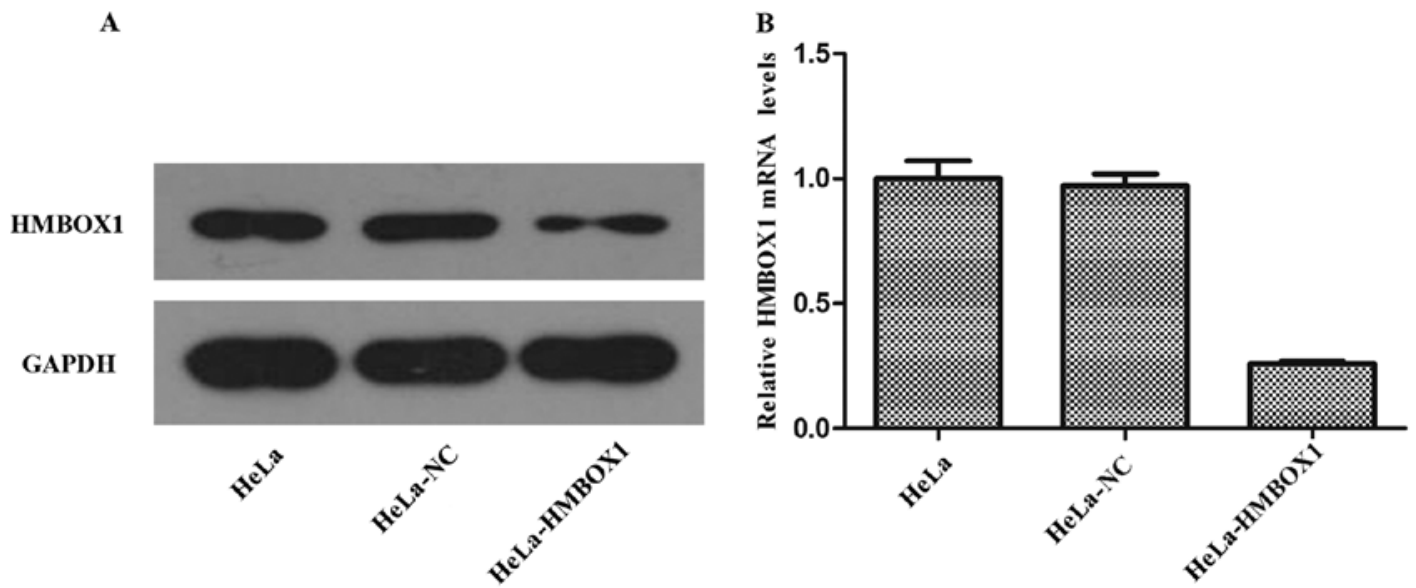

Figure 1. Verification of the efficiency of HMBOX1 knockdown by western blotting and real-time PCR. (A) Western blot analysis showed that the protein level of HMBOX1 in the HeLa-HMBOX1 cells was decreased as compared to the levels in the HeLa and HeLa-NC cells. (B) The relative mRNA levels were detected by real-time PCR. The histogram shows that the mRNA level in the HeLa-HMBOX1 cells was significantly decreased as compared to the other two groups $(\mathrm{P}<0.05)$.
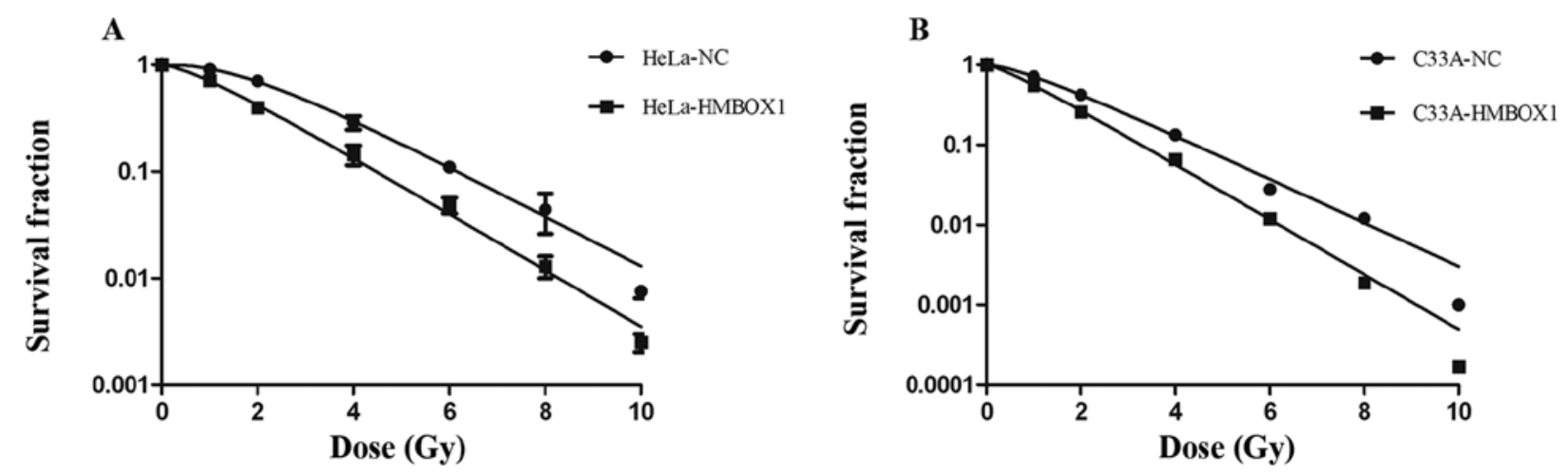

Figure 2. Colony formation assay was used to determine the effect of HMBOX1 knockdown on the radiosensitivity of the HeLa and C33A cells. (A) SF2 (survival fraction after irradiation with $2 \mathrm{~Gy}$ ) of the HeLa-NC and HeLa-HMBOX1 cells was $0.71 \pm 0.04$ and $0.40 \pm 0.07$, respectively $(\mathrm{P}<0.05)$. (B) SF2 of C33A-NC and C33A-HMBOX1 cells was $0.40 \pm 0.04$ and $0.23 \pm 0.03(\mathrm{P}<0.05)$.

Knockdown of HMBOXI increases the radiosensitivity of HeLa and C33A cells. Colony formation assay is a cell survival assay that reflects the ability to grow into a colony of cells. The survival fraction after irradiation with 2 Gy X-rays (SF2) is a main target in assessing the radiosensitivity of cancer cells. Compared with the negative control cells, the knockdown cells had a significantly lower SF2 (Fig. 2A and B). The knockdown cell lines showed higher radiosensitivity compared with that noted in the negative control cell lines.

Knockdown of HMBOX1 increases spontaneous and radiation-induced apoptosis. Flow cytometry was used to evaluate the effect of HMBOX1 knockdown on the apoptosis rate in HeLa cells. The results of flow cytometry showed that when compared with the negative control groups, there was an increase in the apoptosis rate both in the HeLa-HMBOX1 cell line with or without IR exposure (Fig. 3A and B). The expression levels of apoptosis-related protein caspase-3 were consistent with the results of the flow cytometry (Fig. 3C).

Knockdown of HMBOX1 reduces the ability to repair DNA damage induced by irradiation. To investigate the effect of HMBOX1 knockdown on the DNA damage response, we used immunofluorescence assay to detect DNA damage foci, and found that HMBOX1 knockdown significantly decreased radiation-induced DNA damage foci $(\gamma \mathrm{H} 2 \mathrm{AX})$ compared with the negative control group after irradiation. Furthermore, there was no difference between the two cell lines without IR exposure (Fig. 4A). Regarding DNA damage repair proteins, HMBOX1 knockdown significantly reduced the expression of ATM, ATR, p-ATM, p-ATR and BRCA1 (Fig. 4B).

Knockdown of HMBOXI shortens telomere length through TERT. As shown in Fig. 5B, we verified that knockdown of HMBOX1 shortened telomere length in HeLa cells in accordance with a previous study (5). Moreover, the expression of TERT was significantly decreased in the HeLa-HMBOX1 cells (Fig. 5A). To investigate the role of TERT in the process of telomere shortening by HMBOX1 knockdown, HeLa-HMBOX1 cells were transfected with pHBLV-CMVIE-ZsGreen-TERT (Fig. 5C and D). TERT overexpression rescued telomere shortening in the HeLa-HMBOX1 cells (Fig. 5E). 

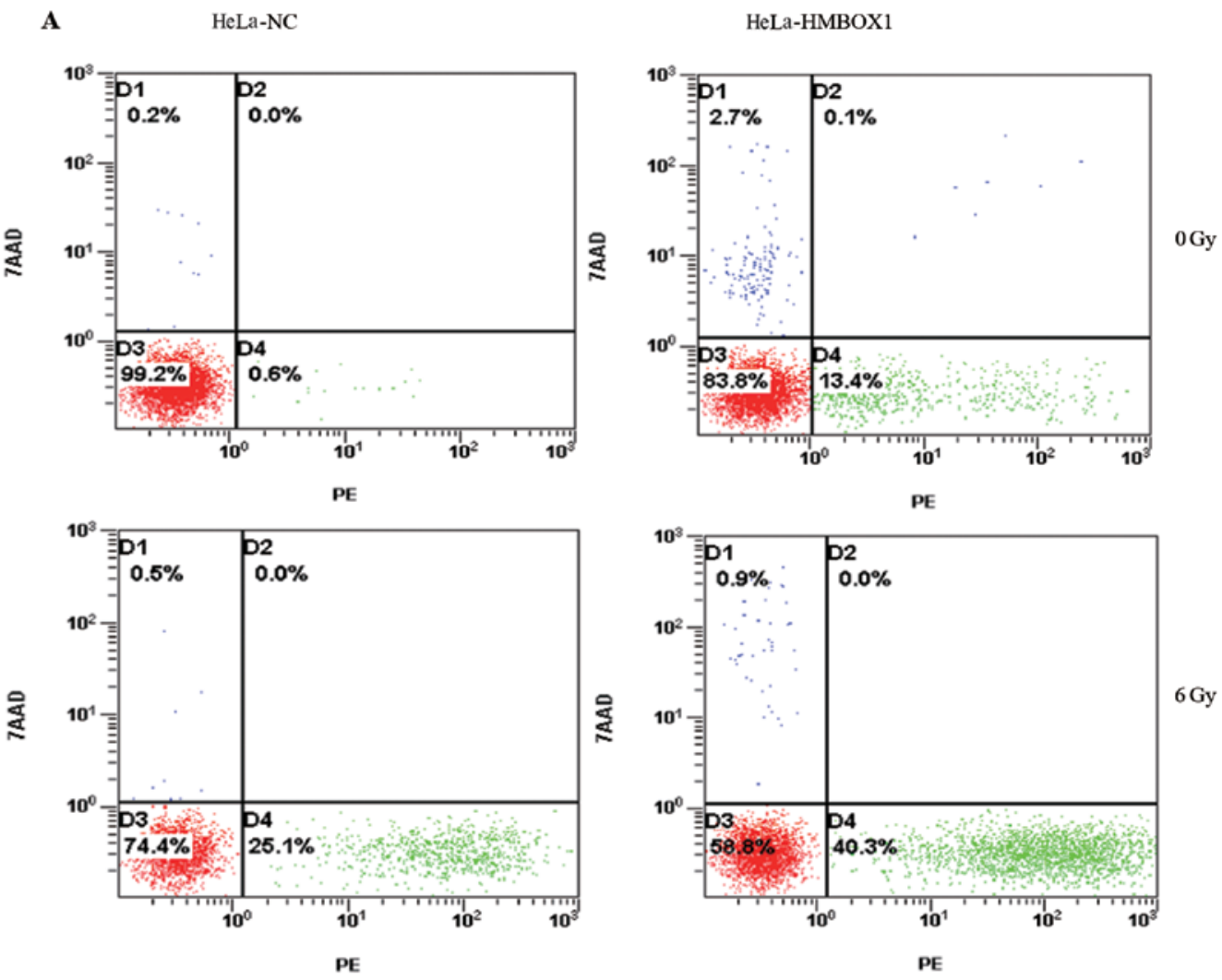

B

C
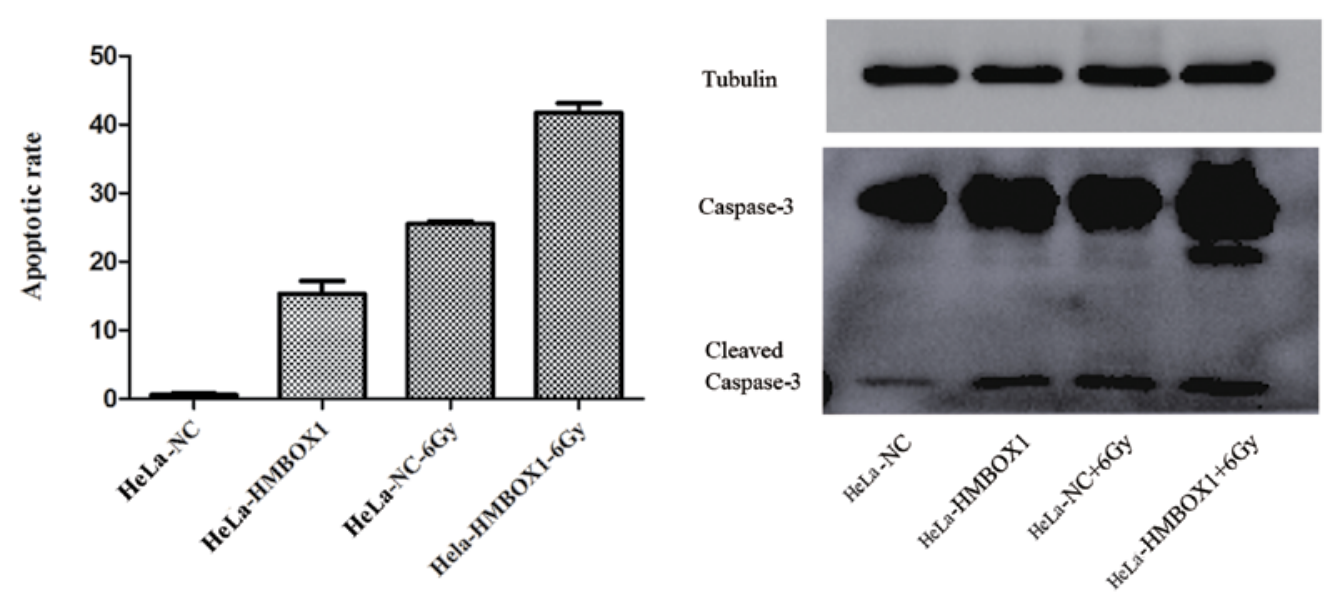

Figure 3. HMBOX1 knockdown increases spontaneous and radiation-induced apoptosis in HeLa cells. (A) Apoptosis rates in the HeLa-NC and HeLa-HMBOX1 cells were $0.6 \pm 0.2$ and $15.3 \pm 1.9 \%$, respectively $(\mathrm{P}<0.05)$. After $6 \mathrm{~Gy}$ X-ray irradiation exposure, apoptosis rates in the two groups were $25.5 \pm 0.4$ and $41.7 \pm 1.4 \%$, respectively $(\mathrm{P}<0.05)$. (B) Histogram shows apoptotis rates of each group. (C) Apoptosis-related protein (caspase-3 cleaved band) increased in the HeLaHMBOX1 and HeLa-HMBOX1-6Gy cells as compared to the HeLa-NC and HeLa-NC-6Gy cells, respectively.

\section{Discussion}

In the present study, we demonstrated that knockdown of HMBOX1 increased the radiosensitivity of cervical cancer HeLa and C33A cells through telomere shortening.

A previous study confirmed that HMBOX1 could directly bind to double-stranded telomeric DNA (4) and participate in telomere length regulation (5). In the present study, we repeated a previous procedure and revealed that knockdown of HMBOX1 could negatively regulate telomere length in HeLa cells. Telomeres play a crucial role in radiotherapy. Telomere length can be used to predict the radiosensitivity of patients with cancers (7). Therefore, we hypothesized that the radiosensitivity of $\mathrm{HeLa}$ and $\mathrm{C} 33 \mathrm{~A}$ cells could be increased by HMBOX1 knockdown through telomere shortening.

To test this hypothesis, we established stable transfected cell lines of HeLa and C33A. We found that HMBOX1 knockdown shortened telomere length and decreased ATM, ATR, BRCA1 protein expression levels which play a crucial role in DNA damage repair response. Colony formation assay revealed that knockdown of HMBOX1 increased radiosensitivity of the HeLa and C33A cells. These data confirmed that HMBOX1 knockdown was closely related to the radiosensitivity of cervical cancer cells. 


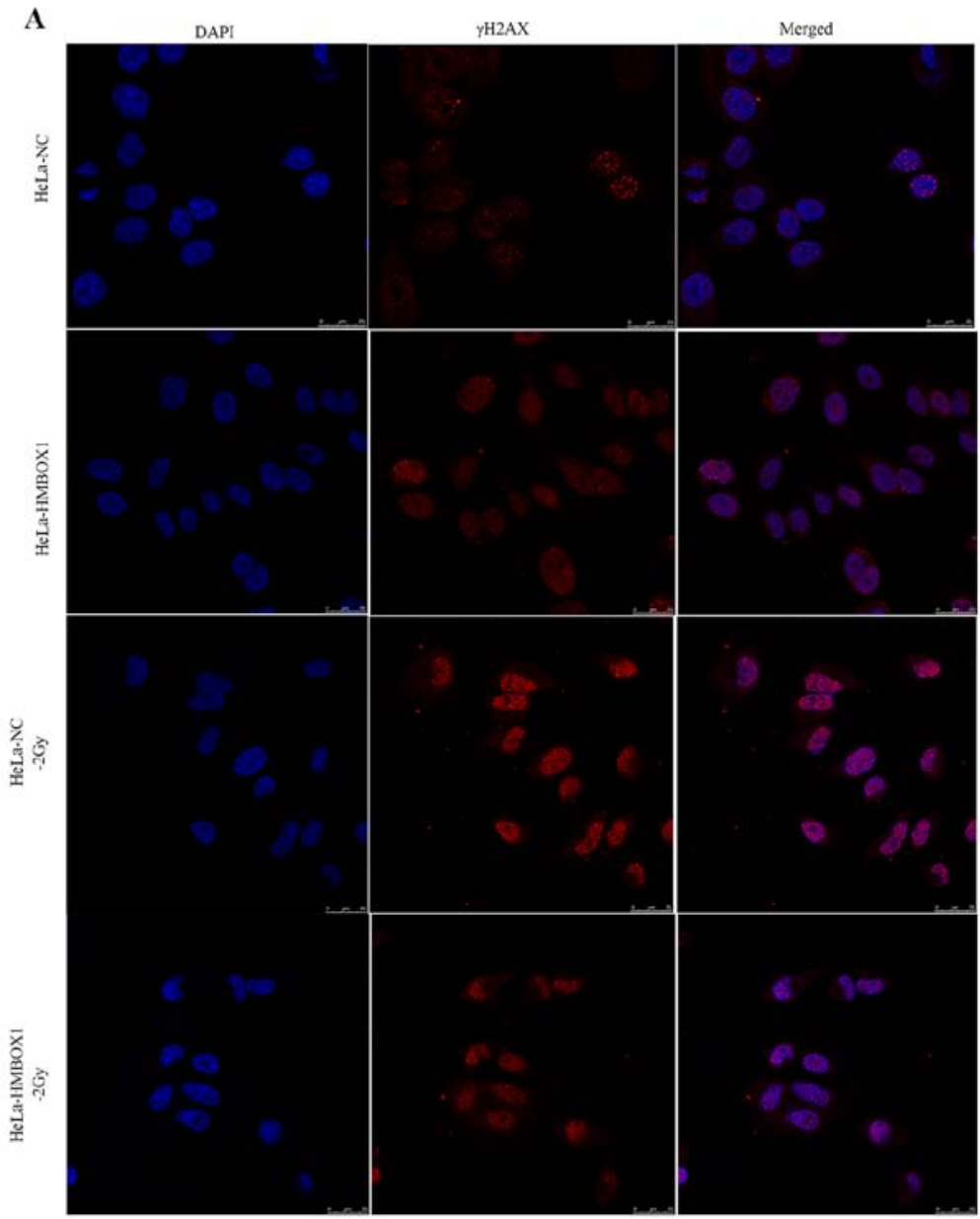

B

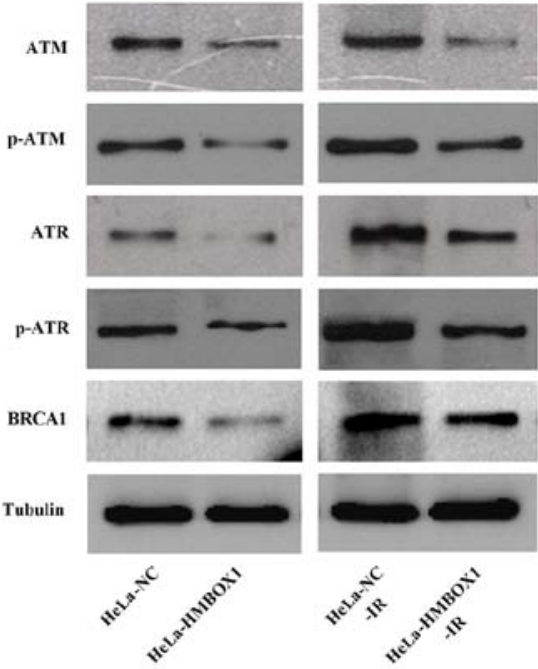

Figure 4. Effects of HMBOX1 knockdown on the ability of DNA damage repair. (A) Without irradiation there was no difference between the two groups. When the cells were exposed to $2 \mathrm{~Gy}$ X-ray and incubated for $30 \mathrm{~min}$, the number of DNA damage foci in the HeLa-HMBOX1 cells were obviously decreased as compared to the HeLa-NC cells. (B) ATM, ATR, p-ATM, p-ATR and BRCA1 proteins were obviously decreased in the HeLa-HMBOX1 cells with or without IR

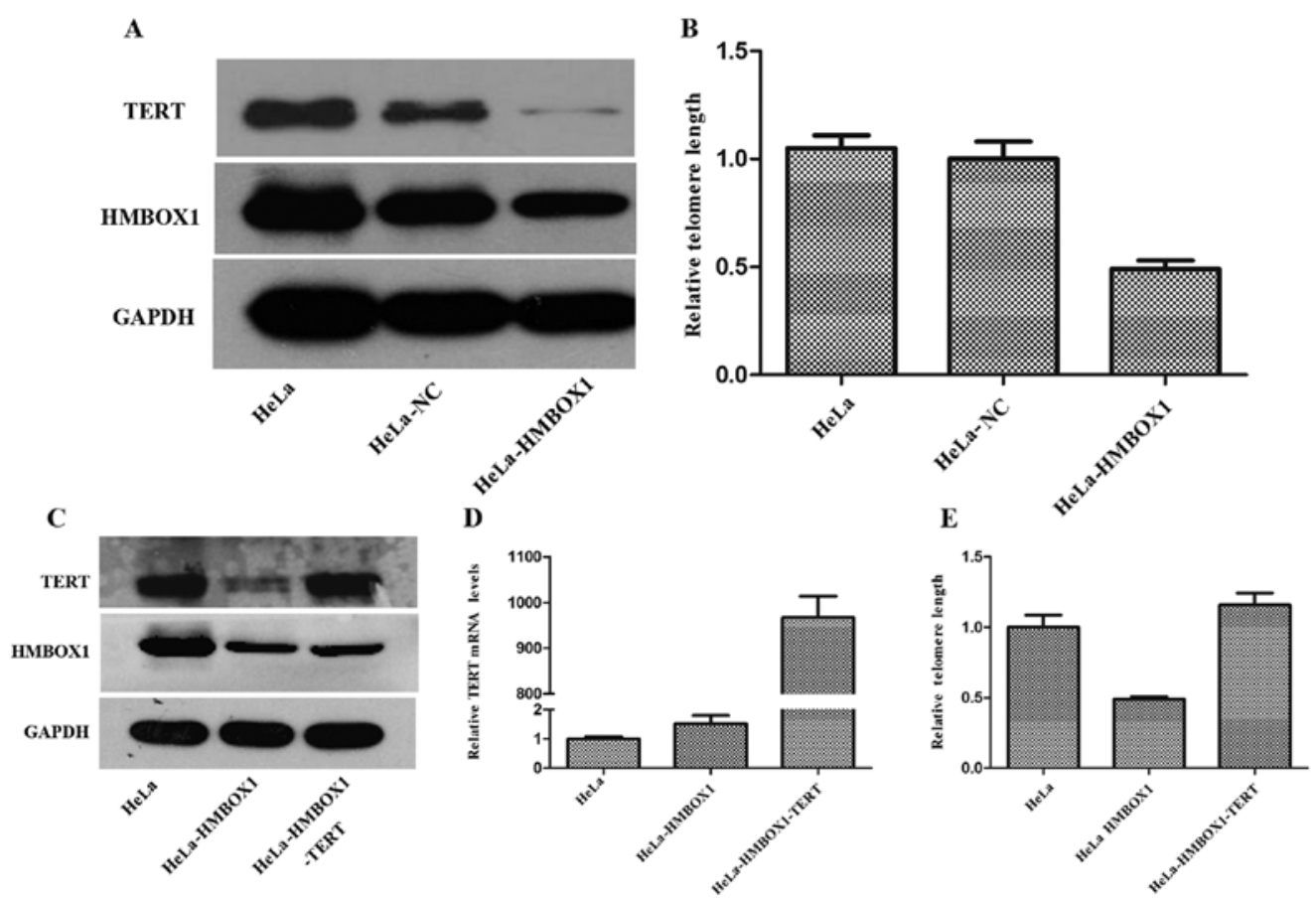

Figure 5. Effects of HMBOX1 knockdown on telomere length. (A) Knockdown of HMBOX1 decreased HMBOX1 protein level. Moreover, TERT protein was also decreased. (B) Knockdown of HMBOX1 shortened telomere length. (C and D) The expression levels of TERT protein and mRNA are shown. (E) TERT overexpression rescued telomere shortening in the HeLa-HMBOX1 cells. 
There is scarce research referring to the relationship between HMBOX1 and the DNA damage repair response. Immunofluorescence showed that HMBOX1 knockdown decreased $\gamma \mathrm{H} 2 \mathrm{AX}$ induced by irradiation which was identified as a molecular marker of DSBs (16). After IR exposure, $\mathrm{H} 2 \mathrm{AX}$ becomes phosphorylated within a few minutes and forms foci at DNA break sites. $\gamma \mathrm{H} 2 \mathrm{AX}$ plays a critical role in the recruitment of repair factors to nuclear foci after DNA damage such as Rad50, Rad51 and BRCA1. Moreover, the process facilitates further recruitment of other DNA damage response factors (PI-3 protein family, p53 and NBS1) to DNA break sites (7,17-19).

In mammalian cells, nonhomologous end joining (NHEJ), and homologous recombination (HR) are primary repair pathways in DNA DSBs. ATM and ATR protein kinase are the main upstream checkpoint kinases in the HR pathway, while BRCA1 is an important downstream protein. Radiationinduced DNA damage contains double- and single-stranded breaks. The ATM pathway is thought to respond primarily to double-stranded breaks, whereas ATR is primarily required in single-stranded breaks (16). We found that knockdown of HMBOX1 decreased the expression of ATM, ATR and BRCA1 with or without ionizing radiation exposure, as well as phospho-ATM and phospho-ATR. Previous studies have verified that ATM, and ATR expression levels are closely related to radiosensitivity in cancer cells (20-24), and inhibition of radiation-induced DNA damage repair is believed to lead to radiosensitization (25). Our data indicate that knockdown of HMBOX1 resulted in lower expression of ATM, ATR and BRCA1 in the HR pathway.

Previous studies and our studies have demonstrated that knockdown of HMBOX1 leads to telomere shortening. Telomere attrition serves as a suppression mechanism of tumors, and suppresses the proliferative capacity of cells resulting in the suppression of cell clone growth (7). Short telomeres contain less shelterin complex that contributes to telomere maintenance. Telomere attrition may lead to telomere dysfunction due to the failing of shelterins to adhere to short telomeres $(7,26)$. Telomere shortening directly contributes to the DNA damage, which leads to chromosomal breaks, while chromatin structure influences DNA repair process in turn $(16,19)$. In U2OS cells, knockdown of HMBOX1 increased both telomere dysfunction-induced foci (TIFs)/cell and the percentage of TIF-positive cells (6). Although we did not repeat the experiment, we believe that knockdown of HMBOX1 has the same effect in HeLa cells based on this evidence. Thus, we proposed that knockdown of HMBOX1 may result in the DNA damage response through telomere shortening, which may be a main mechanism of radiosensitization.

Telomerase reverse transcriptase (TERT) and telomerase RNA component (TERC) are the two core elements of telomerase which works as a ribonucleoprotein complex. TERT serves as an enzyme with reverse transcription activity that is essential for adding telomeric repeats to the chromosome end (5). Previous studies have verified that there is a negative correlation between TERT and the radiosensitivity of cancer cells $(11,14)$. We found that expression of TERT also decreased while HMBOX1 was downregulated. Thus, TERT may act as a mediator between HMBOX1 and telomere length. Notably, overexpression of TERT in HeLa-HMBOX1 cells rescued telomere shortening, which provided forceful evidence of the link between HMBOX1 and telomere length. However, knockdown of HMBOX1 in U2OS cells (ALT cell) did not influence telomere length, which indirectly confirmed that TERT plays a crucial role in the process of the regulation and telomere length by HMBOX1.

Ma et al showed that knockdown of HMBOX1 induced apoptosis in vascular endothelial cells in mice (27). We found that HMBOX1 had the same effect in HeLa cells. Moreover, HeLa-HMBOX1 cells showed a significantly higher apoptosis rate compared with HeLa-NC cells after ionizing radiation exposure. Furthermore, telomere shortening induced p53-dependent and -independent apoptosis $(28,29)$. Previous studies have verified that endothelial cell apoptosis and antiangiogenic therapy enhance the tumor response to radiotherapy (30-32). Combined effects of antiangiogenic and HMBOX1 knockdown in radiotherapy need further investigation. These results indicated that increasing spontaneous and radiation-induced apoptosis via telomere shortening may contribute to radiosensitization.

The main aim of the present study was to assess the relationship between HMBOX1 and radiosensitivity. The present study verified that knockdown of HMBOX1 increased radiosensitivity through telomere shortening in cervical cancer cells. We demonstrated the correlation among HMBOX1, telomere length and TERT. Furthermore, the present study enhanced our understanding of the link between DNA damage repair response and HMBOX1. Finally, the combination of HMBOX1 knockdown and ionizing radiation may have a synergistic effect on apoptosis. Together, our data suggest a model in which HMBOX1 knockdown shortens telomere length that limits repair of DNA damage and induces cell apoptosis, thereby providing a reasonable explanation for the increased radiosensitivity of cervical cancer cells.

\section{Acknowledgements}

The authors thank Sulin Mi for the editorial assistance.

\section{References}

1. Torre LA, Bray F, Siegel RL, Ferlay J, Lortet-Tieulent J and Jemal A: Global cancer statistics, 2012. CA Cancer J Clin 65: 87-108, 2015

2. Zuliani AC, Esteves SC, Teixeira LC, Teixeira JC, de Souza GA and Sarian LO: Concomitant cisplatin plus radiotherapy and high-dose-rate brachytherapy versus radiotherapy alone for stage IIIB epidermoid cervical cancer: A randomized controlled trial. J Clin Oncol 32: 542-547, 2014.

3. Chen S, Saiyin H, Zeng X, Xi J, Liu X, Li X and Yu L: Isolation and functional analysis of human HMBOX1, a homeobox containing protein with transcriptional repressor activity. Cytogenet Genome Res 114: 131-136, 2006.

4. Déjardin J and Kingston RE: Purification of proteins associated with specific genomic Loci. Cell 136: 175-186, 2009.

5. Kappei D, Butter F, Benda C, Scheibe M, Draškovič I, Stevense M, Novo CL, Basquin C, Araki M, Araki K, et al: HOT1 is a mammalian direct telomere repeat-binding protein contributing to telomerase recruitment. EMBO J 32: 1681-1701, 2013.

6. Feng X, Luo Z, Jiang S, Li F, Han X, Hu Y, Wang D, Zhao Y, Ma W, Liu D, et al: The telomere-associated homeoboxcontaining protein TAH1/HMBOX1 participates in telomere maintenance in ALT cells. J Cell Sci 126: 3982-3989, 2013.

7. Palm W and de Lange T: How shelterin protects mammalian telomeres. Annu Rev Genet 42: 301-334, 2008. 
8. de Lange T: Protection of mammalian telomeres. Oncogene 21: 532-540, 2002.

9. Blackburn EH: Switching and signaling at the telomere. Cell 106: 661-673, 2001.

10. McIlrath J, Bouffler SD, Samper E, Cuthbert A, Wojcik A, Szumiel I, Bryant PE, Riches AC, Thompson A, Blasco MA, et al: Telomere length abnormalities in mammalian radiosensitive cells. Cancer Res 61: 912-915, 2001.

11. Mirjolet C, Boidot R, Saliques S, Ghiringhelli F, Maingon P and Créhange G: The role of telomeres in predicting individual radiosensitivity of patients with cancer in the era of personalized radiotherapy. Cancer Treat Rev 41: 354-360, 2015.

12. Yang $\mathrm{H}$, Wu L, Ke S, Wang W, Yang L, Gao X, Fang H, Yu H, Zhong Y, Xie C, et al: Downregulation of ubiquitin-conjugating enzyme UBE2D3 promotes telomere maintenance and radioresistance of Eca-109 human esophageal carcinoma cells. J Cancer 7: 1152-1162, 2016.

13. Cawthon RM: Telomere measurement by quantitative PCR. Nucleic Acids Res 30: e47, 2002.

14. Gao X, Wang W, Yang H, Wu L, He Z, Zhou S, Zhao H, Fu Z, Zhou F and Zhou Y: UBE2D3 gene overexpression increases radiosensitivity of EC109 esophageal cancer cells in vitro and in vivo. Oncotarget 7: 32543-32553, 2016.

15. Franken NA, Rodermond HM, Stap J, Haveman J and van Bree C: Clonogenic assay of cells in vitro. Nat Protoc 1: 2315-2319, 2006.

16. d'Adda di Fagagna F, Reaper PM, Clay-Farrace L, Fiegler H, Carr P, Von Zglinicki T, Saretzki G, Carter NP and Jackson SP: A DNA damage checkpoint response in telomere-initiated senescence. Nature 426: 194-198, 2003.

17. Paull TT, Rogakou EP, Yamazaki V, Kirchgessner CU, Gellert M and Bonner WM: A critical role for histone $\mathrm{H} 2 \mathrm{AX}$ in recruitment of repair factors to nuclear foci after DNA damage. Curr Biol 10: 886-895, 2000.

18. Shiloh Y: ATM and related protein kinases: Safeguarding genome integrity. Nat Rev Cancer 3: 155-168, 2003

19. Drissi R, Wu J, Hu Y, Bockhold C and Dome JS: Telomere shortening alters the kinetics of the DNA damage response after ionizing radiation in human cells. Cancer Prev Res 4: 1973-1981, 2011.

20. Yang L, Wang W, Hu L, Yang X, Zhong J, Li Z, Yang H, Lei H, Yu H, Liao Z, et al: Telomere-binding protein TPP1 modulates telomere homeostasis and confers radioresistance to human colorectal cancer cells. PLoS One 8: e81034, 2013.
21. Tribius S, Pidel A and Casper D: ATM protein expression correlates with radioresistance in primary glioblastoma cells in culture. Int J Radiat Oncol Biol Phys 50: 511-523, 2001.

22. Rainey MD, Charlton ME, Stanton RV and Kastan MB: Transient inhibition of ATM kinase is sufficient to enhance cellular sensitivity to ionizing radiation. Cancer Res 68: 7466-7474, 2008.

23. Chang L, Graham PH, Hao J, Ni J, Bucci J, Cozzi PJ, Kearsley JH and $\mathrm{Li} \mathrm{Y}: \mathrm{PI} 3 \mathrm{~K} / \mathrm{Akt} / \mathrm{mTOR}$ pathway inhibitors enhance radiosensitivity in radioresistant prostate cancer cells through inducing apoptosis, reducing autophagy, suppressing NHEJ and HR repair pathways. Cell Death Dis 5: e1437, 2014.

24. Alao JP and Sunnerhagen P: The ATM and ATR inhibitors CGK733 and caffeine suppress cyclin D1 levels and inhibit cell proliferation. Radiat Oncol 4: 51, 2009.

25. Zhu WG, Seno JD, Beck BD and Dynlacht JR: Translocation of MRE11 from the nucleus to the cytoplasm as a mechanism of radiosensitization by heat. Radiat Res 156: 95-102, 2001.

26. Loayza D and De Lange T: POT1 as a terminal transducer of TRF1 telomere length control. Nature 423: 1013-1018, 2003.

27. Ma H, Su L, Yue H, Yin X, Zhao J, Zhang S, Kung H, Xu Z and Miao J: HMBOX1 interacts with MT2A to regulate autophagy and apoptosis in vascular endothelial cells. Sci Rep 5: 15121, 2015.

28. Ribero S, Sanna M, Visconti A, Navarini A, Aviv A, Glass D, Spector TD, Smith C, Simpson M, Barker J, et al: Acne and telomere length: A new spectrum between senescence and apoptosis pathways. J Invest Dermatol 137: 513-515, 2017.

29. Wang Y, Wang X, Flores ER, Yu J and Chang S: Dysfunctional telomeres induce p53-dependent and independent apoptosis to compromise cellular proliferation and inhibit tumor formation. Aging Cell 15: 646-660, 2016.

30. Mauceri HJ, Hanna NN, Beckett MA, Gorski DH, Staba MJ, Stellato KA, Bigelow K, Heimann R, Gately S, Dhanabal M, et al: Combined effects of angiostatin and ionizing radiation in antitumour therapy. Nature 394: 287-291, 1998.

31. Garcia-Barros M, Paris F, Cordon-Cardo C, Lyden D, Rafii S, Haimovitz-Friedman A, Fuks Z and Kolesnick R: Tumor response to radiotherapy regulated by endothelial cell apoptosis. Science 300: 1155-1159, 2003.

32. Meng MB, Jiang XD, Deng L, Na FF, He JZ, Xue JX, Guo WH, Wen QL, Lan J, Mo XM, et al: Enhanced radioresponse with a novel recombinant human endostatin protein via tumor vasculature remodeling: Experimental and clinical evidence. Radiother Oncol 106: 130-137, 2013. 